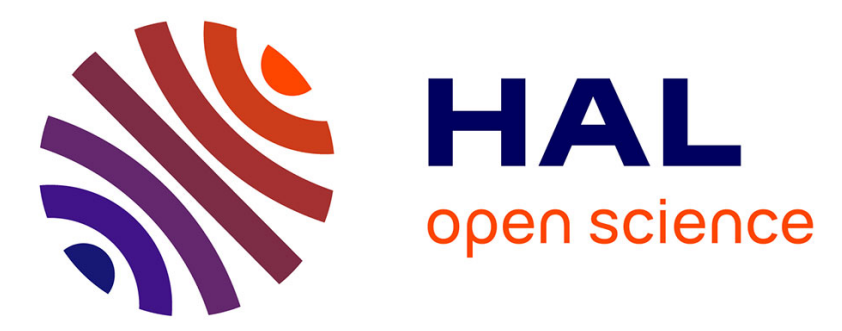

\title{
An Enhanced AODV Protocol for VANETs with Realistic Radio Propagation Model Validation
}

Jonathan Ledy, Hervé Boeglen, Benoit Hilt, Hafid Abouaissa, Rodolphe

Vauzelle

\section{- To cite this version:}

Jonathan Ledy, Hervé Boeglen, Benoit Hilt, Hafid Abouaissa, Rodolphe Vauzelle. An Enhanced AODV Protocol for VANETs with Realistic Radio Propagation Model Validation. ITST 2009, Oct 2009, Lille, France. pp.5. hal-00476738

\section{HAL Id: hal-00476738 \\ https://hal.science/hal-00476738}

Submitted on 27 Apr 2010

HAL is a multi-disciplinary open access archive for the deposit and dissemination of scientific research documents, whether they are published or not. The documents may come from teaching and research institutions in France or abroad, or from public or private research centers.
L'archive ouverte pluridisciplinaire HAL, est destinée au dépôt et à la diffusion de documents scientifiques de niveau recherche, publiés ou non, émanant des établissements d'enseignement et de recherche français ou étrangers, des laboratoires publics ou privés.

$$
\text { Copyright }
$$




\title{
An Enhanced AODV Protocol for VANETs with Realistic Radio Propagation Model Validation
}

\author{
Jonathan LEDY ${ }^{1}$, Hervé BOEGLEN ${ }^{1}$, Benoît HILT ${ }^{1}$ \\ Abdelhafid ABOUAISSA ${ }^{1}$, Rodolphe VAUZELLE ${ }^{2}$
}

\author{
${ }^{1}$ Laboratoire MIPS/GRTC \\ Université de Haute Alsace, France \\ [jonathan.ledy, herve.boeglen, benoit.hilt, \\ abdelhafid.abouaissa]@uha.fr
}

\author{
${ }^{2}$ Laboratoire XLIM/SIC \\ Université de Poitiers, France \\ vauzelle@sic.sp2mi.univ-poitiers.fr
}

\begin{abstract}
In this paper we evaluate V-AODV a version of AODV (Ad-hoc On-demand Distance Vector) especially created for Vehicular Ad-hoc NETworks (VANETs). V-AODV is designed to run with a complex cross layered metric based on both delay from node to node and Bit Error Rate (BER) coming from the physical layer. We conducted simulations with the NS2 simulator taking in account a realistic environment tool called Communication Ray Tracer (CRT). Our results show that the basic propagation models usually in use with NS2 are not suitable for VANETs simulations. We also show that when using a routing metric based on delay and BER, the first parameter is more relevant in terms of QoS than the second one.
\end{abstract}

Index Terms - VANETs, QoS, Realistic Propagation Models, AODV

\section{INTRODUCTION}

One of the most promising applications of Ad-Hoc networking technology is certainly the Vehicular Ad-hoc NETs (VANETs). VANETs are not only suitable for commercial and entertainment applications but also for safety and traffic management. In those fields, one of the main concerns is Quality of Service (QoS). Safety information must be carried in a highly robust manner in networks which are self-organizing and characterized by limited degrees of freedom in node movements and high speed variations. This situation leads to a network topology changing very frequently and possibly very fast. Many research and development works relating to routing [1], communication robustness [2], information dissemination [3] take this point into account and show results obtained with simulations involving very simplistic radio propagation models available in simulation tools.
However, frequent topology changes involve, especially in urban or high density zones, highly variable quality of the underlying radio channel. To the best of our knowledge, only a few number of papers [4][5][6] relate to the effects in simulations of the radio propagation models. Moreover, only one [4] takes into account the $802.11 \mathrm{p}$ enhancements to Intelligent Transportation Systems. These papers indicate that the more realistic the propagation model, the more the percentage of received messages decreases.

When VANETs carry safety information, QoS is of a special importance in delivering messages and especially in the end to end parameter. For some other applications like communications tools or some entertainment applications, QoS can be needed too.

But if there is no satisfying radio link, which can be seen as a common resource in a given neighborhood, no communication can take place. Fading, time variation, nodes mobility and multi-path effects lead to frequent broken routes, especially in the presence of high mobility nodes. Consequently, providing end-to-end QoS support guarantee is even more challenging, because in a high speed mobility context, many data packets are lost and the failure notifications together with the overhead due to route repairs increase significantly.

To bring these two points together, we propose $\mathrm{V}$ AODV, an enhancement to the AODV routing protocol for VANETs. V-AODV can be seen as a cross layer running between the PHY and the Network layers. In VAODV we use a metric based on the radio link quality information combined with delay information which is, as previously discussed, most relevant in the propagation of safety messages. Another important point of this work is the use of a realistic propagation model. 
Taking into account of these elements in NS2 based simulations, allows us to validate our strategy by showing how our new metric improves the end to end delay and packet loss in realistic conditions.

The rest of this paper is organized as follows. Section II presents the realistic channel model we use for validation of the proposed enhanced routing protocol. In Section III we describe the enhancements brought by the $\mathrm{V}$-AODV routing protocol. Section IV is devoted to the performance evaluation of V-AODV. Finally Section V concludes the paper and highlights ideas for future work.

\section{A REALISTIC PROPAGATION MODEL FOR VANETS}

Many researchers in the field of wireless networking use the network simulator NS2 for the evaluation and validation of their routing protocols. However, it is known that NS2 does not model accurately the radio propagation channel [4]. In fact, NS2 Radio Propagation Models (RPM) do not allow specifying complex environments as such containing obstacles. This feature is however a crucial issue in the context of VANETs. Besides the very simplistic RPMs implemented in NS2, the error model used by this network simulator is also an important issue. In fact, the native NS2 error model only checks if the received power of a packet is above or below the receiver's threshold.

In order to overcome the above mentioned limitations we have integrated into NS2 a realistic RPM called Communication Ray Tracer (CRT) which has been developed at the SIC-XLIM laboratory [7]. This RPM is based on a ray-tracing method and allows to model very accurately the propagation mechanisms involved in a realistic environment described by a scene like the one shown on Figure 1.

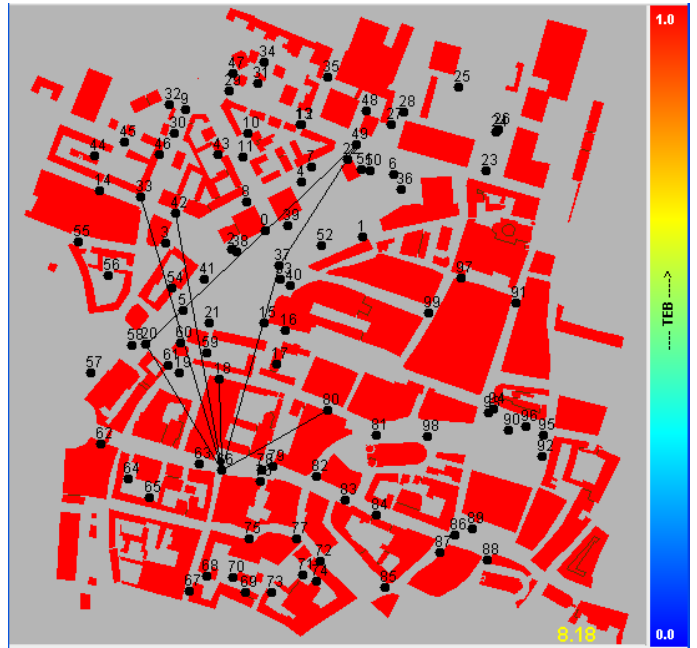

Fig. 1: Vehicles moving in the Munich city center
An appropriate error model according to the $802.11 \mathrm{p}$ standard has also been implemented in CRT. This error model is based on the calculation of the Bit Error Rate (BER) for each link. In order to obtain the BER between two nodes, the whole $802.11 \mathrm{p}$ standard physical layer has been implemented. The Signal to Noise Ratio (SNR) is then used as an entry parameter for BER calculation. The SNR is derived from the received power provided by CRT and the receiver noise floor according to equation (1). From the BER value, the Packet Error Rate (PER) of each link is finally obtained by using equation (2).

$$
\begin{gathered}
\mathrm{SNR}=10 \log (\text { RPower_/Noise_ }) \\
\text { PER }=1-(1-\mathrm{BER})^{\mathrm{N}}
\end{gathered}
$$

where RPower_ is the packet signal strength at the receiver, and $\mathrm{N}$ represents the packet number of bits. Finally, the BER parameter is added to every packet transmitted and is therefore available to the upper layers of the network.

\section{V-AODV, QOS IN AODV ROUTING PROTOCOL}

The insufficient resources in VANETs have made the QoS routing strategy a challenging task. To overcome this problem, a simple and efficient routing mechanism is required to manage the resource requests, while at the same time it has to be adaptable to the frequent changing topology conditions.

Since communications in VANET should support at least two kinds of messages, i.e. Comfort and Alert messages, the QoS routing needs to provide at least two levels of QoS to applications. The first level deals with released parameters and the second with strained parameters. In the case of the latter level, a scheme is required to provide connection control to new flows by predicting their impact on the QoS of the flows already running in the network.

A VANET can be modelled as an undirected graph $G(V, E)$, where $V$ is the set of nodes, and $E$ is the set of links. Motivated by the coloured sub-graphs formulation presented in [8], we divide each link to sub-links represented by elementary cost function for QoS metrics (i.e.: bandwidth, delay, energy, bit error rate (BER), packet loss probability, security, etc.) In this paper, we only focus on delay and BER parameters. In the near future, we plan to examine other metrics like delay jitter, packet loss probability, and security.

Estimating end-to-end delay in a VANET is very difficult due to the unsynchronized nature of the network. In V-AODV we use the "Hello" messages to estimate the delay needed to reach each neighbour. By 
mean of this, we make available a cross matrix of the network topology containing each node to neighbour delays. These calculated delays represent the Round Trip Time (RTT) between the node originator of the Hello message and their neighbours returning acknowledgements. Thus, the delay cost function of a given node "i" can be represented as follow:

$$
c_{d}=\frac{D_{i, j}}{D_{\max }-\sum_{i=1}^{j} D_{i}}
$$

where:

$D_{i, j}:$ is the estimated delay to the next hop,

$\mathrm{D}_{\max }$ : means the limit bound of delay supported by a flow,

$\sum_{i} D$ : represents the accumulated delay from the source "i" to the node " $\mathrm{j}$ ".

The BER of all moving links in the network are obtained by using the CRT RPM together with the error model presented in section II.

The BER cost function is the given by:

$$
c_{B E R}=\frac{1}{B E R_{\max }-B E R_{\text {estimated }}}
$$

Specifically, the local cost functions $C B E R$ and $C d$ are hyperbolic functions limited respectively by BERmax and Dmax. The end-to-end cost of a route is represented by a global function called $\mathrm{C}_{\mathrm{ETE}}$ which results from the sum of cost functions $(C B E R+C d)$ and is evaluated at each node participating in the route discovery process. The value of $\mathrm{C}_{\mathrm{ETE}}$ is accumulated from source to destination. In particular, a high value of $\mathrm{C}_{\mathrm{ETE}}$ means an unacceptable route.

$$
C_{E T E}=\frac{1}{B E R_{\max }-B E R_{\text {estimated }}}+\frac{D_{i, j}}{D_{\max }-\sum_{i=1}^{j} D_{i}}
$$

When a source node wishes to establish a route to another node with respect to the QoS requirement, it disseminates a Route Request (RReq) packet that includes mainly the requested delay but also the end-toend cost function $\mathrm{C}_{\mathrm{ETE}}$ which will be accumulated at every participating node to establish this route.

Upon receiving the route request packet, every intermediate node tries to respond to the QoS requirement by verifying equations (3) and (4). If there is enough resource, the node updates the end-to-end cost (as expressed by (5)) and forwards the RReq to its neighbours until the message reaches the destination.
The latter responds by unicasting a Route Reply packet (RRep) with the $\mathrm{C}_{\mathrm{ETE}}$ cost function to the source along the reverse route.

When the source receives the RouteReply, it selects the optimal route with respect to the smaller value of the cost function $\mathrm{C}_{\mathrm{ETE}}$.

\section{EVALUATION OF V-AODV}

The CRT RPM integrated into NS2 allows us to use either NS2 built-in free space propagation environment or realistic propagation environments and, when needed, to compute the BER between two nodes. All simulations were conducted in an 1122 square meter area representing the core of the city of Munich (Germany) with buildings and streets. In this area 10 mobile nodes are moving. The nodes motion speed is set to $4 \mathrm{~m} / \mathrm{s}$. Hello messages are sent approximately every second by all nodes. During the simulation three communications are scheduled. These communications, which are video flows, take place in a first simulation set between randomly chosen nodes and in a second set between fixed nodes. The data rate of these video flows is set to a $6 \mathrm{Mb} / \mathrm{s}$ rate.

As indicated above, the main measured parameter is the Averaged End to End Delay (AEED). To show the efficiency of our proposition we calculate two other parameters namely the Packet Delivery Ratio (PDR) and the Normalized Overhead Load (NOL) respectively defined as the ratio of the number of successfully delivered packets to those generated by the sources as shown in (6) and as the ratio between the total number of routing packets to the total number of successfully delivered packets as shown in (7). For this last parameter, the Routing Packets include all the packets involved in both route discovery and maintenance (i.e. Hello, RReq and RRep).

$$
\begin{aligned}
& P D R=\frac{\text { Number of succesfully delivered packets }}{\text { Total number of transmitted packets }} \\
& N O L=\frac{\text { Total number of routing packets }}{\text { Number of succesfully delivered packets }}
\end{aligned}
$$

From the data delivery point of view, a higher PDR indicates a lower packet loss rate and therefore a most efficient routing protocol. In real time communications, the routing protocol with higher PDR may not be considered better than the one with lower PDR, since packets which arrive late could be useless although they 
reach the destination successfully. Real time traffic is delay sensitive.

Because the proposed V-AODV protocol can use a metric based on delay, BER or both for routing computation, in the rest of this paper we call V$\mathrm{AODV}_{\text {delay }}$ the first, V-AODV $\mathrm{BER}_{\text {B }}$ the second and V$A O D V_{\text {delay+BER }}$ the combination of the two. The standard AODV is called AODV ${ }_{\text {Hор. }}$

In a first simulation set, we compare the standard AODV protocol to the three versions of V-AODV (i.e. delay, BER and delay+BER) in a free space and in a realistic propagation environment.

If we now take a look at both the AEED (Fig. 2) and the PDR (Fig. 3), we can see that in the free space environment, both AODV and V-AODV $\mathrm{Velay}_{\text {protocols }}$ lead to always possible communications (AEED of 0,003 s and PDR of $100 \%$ ). In the realistic propagation environment we can see that the introduced constraints lead to a growth for $\mathrm{AODV}_{\mathrm{Hop}}$ of the AEED to $0,4 \mathrm{~s}$ and, because of more important packets rejection, a decrease of the PDR to $80 \%$.

When using V-AODV $V_{\text {delay }}$ the additional delay constraint when establishing routes lowers the PDR to $58 \%$. But in the same time, the AEED goes down to $0,220 \mathrm{~s}$, indicating better communication conditions.

With V-AODV $\mathrm{BER}_{\mathrm{B}}$ we observe that both the PDR and the AEED stay at values near to the ones of AODV $\mathrm{Hop}_{\text {. }}$ This means that, in the case of $\mathrm{AODV}_{\mathrm{Hop}}$, when a link is used for a communication between two nodes, its BER has a good value. Only a few hops are discarded due to bad BER.

If we now use a metric taking into account both the delay and BER, we observe that the first results are confirmed because the AEED goes down to $0,230 \mathrm{~s}$ together with a value of $58 \%$ for the PDR, which is a value similar to the $\mathrm{V}-\mathrm{AODV} \mathrm{V}_{\text {Delay }}$ one.

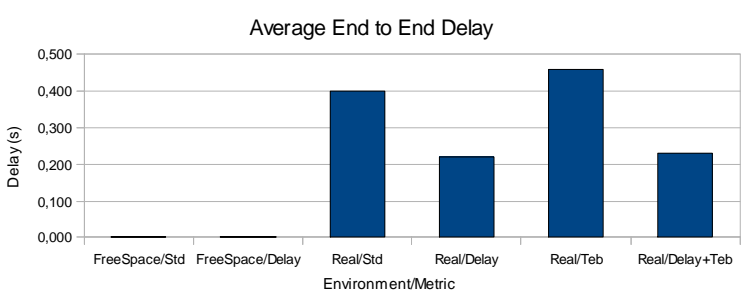

Fig. 2: Average end to end delay

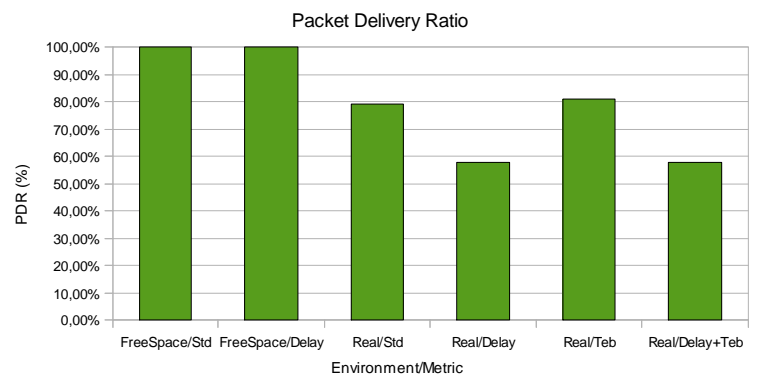

Fig. 3: Packet delivery ratio

In order to get another idea about the efficiency of the protocol, we computed the NOL (Fig. 4). We can see here that in the realistic propagation environment, the propagation constraints lead to an increase of the signaling overhead for $\mathrm{AODV}_{\mathrm{Hop}}$. When using V$\mathrm{AODV}_{\mathrm{BER}}$,in agreement with the AEED and PDR results, the signaling overhead stays in the similar range of values (resp. $11 \%$ and $12 \%$ ).

When the delay parameter is included in the metric, in order to stay under the maximal delay set between a source and a destination, we get more broken routes, leading to a signaling overhead growing from $12 \%$ to $17 \%$.

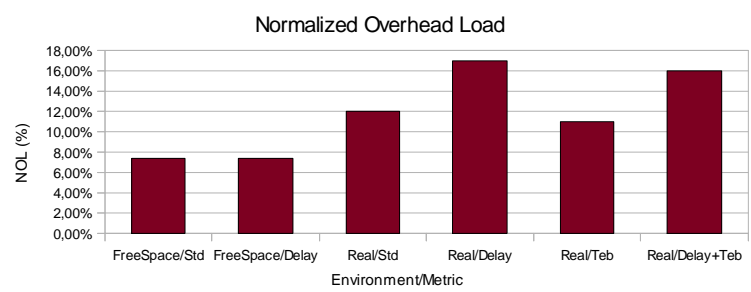

Fig. 4: Normalized overhead load

If we now have a look at the number of dropped packets (Fig. 5), we also observe that the main constraint is related to the delay parameter.

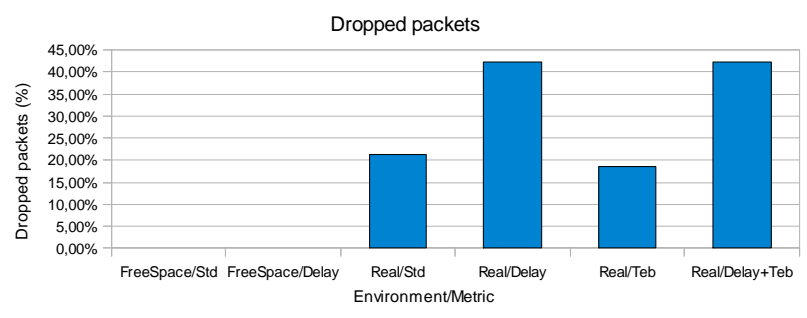

Fig 5: Dropped packets

In order to complete our analysis, Fig. 6 shows that when the routing metric requirements increase, so does the average number of hops. 


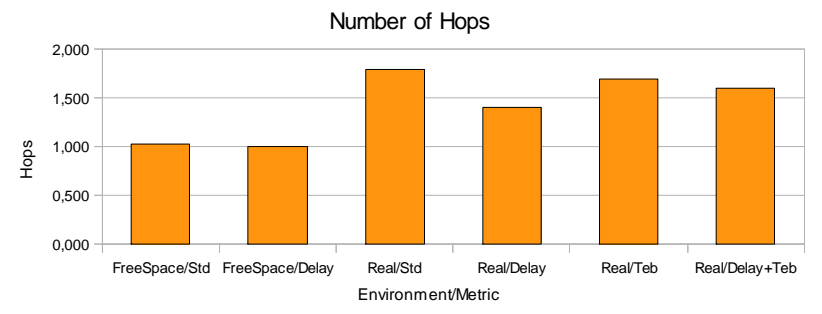

Fig 6: Number of hops

The previous results allow us to conclude that, whatever the propagation model used, when a link between two nodes is suitable for communication, the test of its BER leads to little additional packet rejection. To the contrary, the delay parameter seems to have more importance in route calculation. This appears in each measured indicator. To see how the delay acts on communications, we make additional simulations with fixed couples of sender and receivers. In these simulations we only vary the maximal delay allowed for establishing a communication.

As can be seen in Fig. 7, when the maximal delay decrease, there exists a threshold over which the AEED is constant (over 10ms). At this threshold the AEED decreases in a fast way, so there is no possible communication when it is lower or equal to $0,5 \mathrm{~ms}$.

In Fig. 8 we can see that with a maximal delay of more than $5 \mathrm{~ms}$ multihop communications are allowed while with a $1 \mathrm{~ms}$ delay only one hop communications are possible.

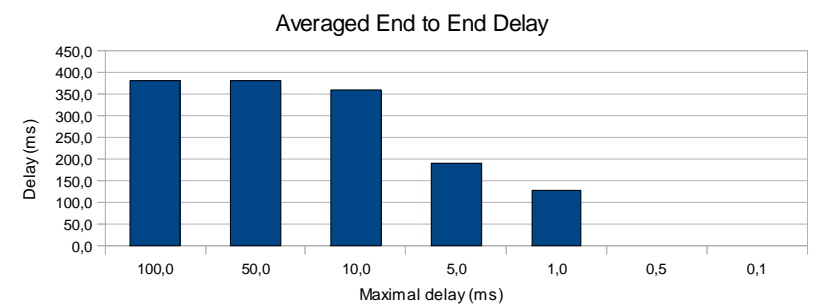

Fig. 7: Averaged End to End Delay

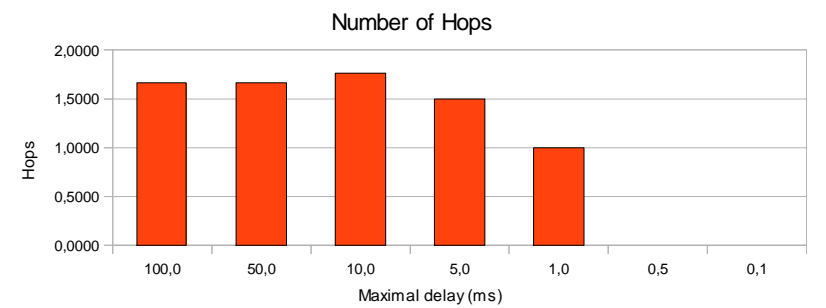

Fig. 8: Number of Hops

We also notice that the maximal delay is computed at the start of a communication and then updated every second. Therefore, due to nodes movements, the measured end to end delay is greater than the maximal delay.
This additional study confirms us that the maximal delay chosen in the first simulation set $(10 \mathrm{~ms})$ is not low enough to prevent communications.

\section{CONCLUSION AND FUTURE WORK}

In this paper we tested an enhanced version of AODV called V-AODV designed for VANETs. The tests were conducted both in a free space propagation environment and in a realistic one.

The first results showed that in the domain of VANETs, simulations must be conducted in realistic environments in order to validate accurately the modification or enhancements applied to a routing protocol.

The evaluation of the V-AODV protocol which routing metric is based on a source to receiver delay measurement and a cross layered physical BER ratio both measured when establishing a route, shows us that the delay parameter takes the precedence over the BER parameter.

We obtained these interesting results in a realistic but limited area. The number of nodes was set to 10 and the number of communications to 3 . Therefore, each time a link between two nodes was available, its BER had a "good" value. In a future work, we will test if in a larger environment, the BER, due to reflections etc... can become a most relevant metric for route calculation.

\section{REFERENCES}

[1] M.Boban, G. Misek, O.Tonguz, "What is best acheivable Qos for Unicast routing in VANET?", GLOBECOM Workshops 2008 IEEE, pp.1-10, Jan. 2008.

[2] S.Wang, "The effects of wireless transmission range on path lifetime in vehicle-formed mobile ad-hoc networks on highways", IEEE International Conference on Communications, vol. 5, pp. 3177-3181, May 2005

[3] S.Yousefi, S.Bastani, M.Fathy, "On the performance of Safety Message Dissemination in Vehicular Ah-hoc Networks", European Conference on Universal Multiservice Networks, 2007. Feb 2007

[4] Francisco J Martinez, Chai-Keong Toh, Juan-Carlos Cano, Carlos T Calafate, Pietro Manzoni, "Realistic Radio Propagation Models (RPMs) for VANET Simulations", IEEE Wireless Communications and Networking Conference, 2009. Mar. 2009.

[5] S.Marinoni, H.H.Kari "Ad-hoc routing protocol performance in a realistic environment", IEEE International Conference on Mobile Communications and Learning Technologies 2006, October 2006.

[6] A.Mahajan, N.Potnis, K.Gopalan, A.Wang, "Modeling vanet deployment in urban settings", International Workshop on Modeling Analysis and Simulation of Wireless and Mobile Systems, 2007, Oct. 2007

[7] F. Mora, L.Aveneau, "Optimized scanning of a visibility graph data structure for efficient ray-tracing", in ECWT2005, Paris, October 2005.

[8] K.M. Konwar, I.I. Mandoiu, A.C. Russell, and A.A. Shvartsman. "Improved Algorithms for Multiplex PCR Primer Set Selection with Amplification Length Constraints", Proc. 3rd Asia-Pacific Bioinformatics Conference (APBC), pp. 41-50, 2005. 